\title{
Unconventional Imaging with Backscattered Electrons
}

\author{
I. Müllerová, Š. Mikmeková, M. Hovorka, and L. Frank
}

Department of Electron Optics, Institute of Scientific Instruments ASCR, v.v.i., Královopolská 147, CZ-61264 Brno, Czech Republic

When imaging with the backscattered electrons (BSE) in a conventional SEM with the sample in the field-free space we acquire the polar angle (PA) of emission between, say, $10^{\circ}$ and $40^{\circ}$ from the surface normal using a ring shaped detector situated below the objective lens (OL) or even a narrower hollow cone of trajectories when a through-the-lens assembly with conversion to secondary electrons is used. In the high resolution SEM the sample is immersed in magnetic field penetrating outside the OL so the BSE follow spiral trajectories and their impact on both types of detectors depends on configuration details. However, still only a relatively narrow interval of PA is acquired and the elastic BSE are usually lost owing to their focusing into the converter bore while at higher energy losses the collection efficiency grows. An immersion electrostatic lens in combination with the OL improves control of the angular selection of BSE [1]. When using the cathode lens (CL) configuration with biased sample [2] we can easily detect both low energy BSE and BSE emitted at large angles with respect to the surface normal even using a below-OL detector, with possible sorting of BSE according to polar angles [3].

It is well known that anisotropy of scattering in solids enhances with decreasing energy of electrons [4]. As regards the angular dependence of the BSE emission from crystals, available models concern the electron backscattering patterns (see e.g. [5]) from which any integral emission data for the single channel detection are difficult to extract. Measurement of the energy/angular distribution of BSE is rather difficult and rarely reported [6] but in practice the capability of acquiring the BSE anisotropy is checked via imaging the grain contrast in polycrystals. Series of experiments performed in the cathode lens mode has confirmed the crystallinity contrast enhanced with keV electrons backscattered at polar angles sufficiently near to $90^{\circ}$ (Figs. 1 and 2). In the range of tens and units of eV, backscattering becomes dependent on electronic structure of the target (Fig. 3) so the crystal orientation may be characterized upon energy dependence of the electron reflectance [7]. At units of eV a kind of the electron beam induced voltage (EBIV) contrast [8] reveals p-type doped patterns in semiconductors (Fig. 4). Further progress in profiting from the complete BSE emission requires introducing a multichannel detection with energy/angular sorting [9].

References

[1] H. Jaksch, Proc. $14^{\text {th }}$ EMC, Aachen 2008, Springer, Berlin, 2008.

[2] I. Müllerová, L. Frank, Adv. Imaging and Electron Phys. 128 (2003) 309.

[3] I. Müllerová, I. Konvalina, L. Frank, Materials Transactions 48 (2007) 940.

[4] L. Reimer, H.G. Badde, H. Seidel, Z. Angew. Phys. 31 (1971) 145.

[5] L. Reimer, Scanning Electron Microscopy, Chapt. 9, Springer, Berlin, 1998.

[6] J. Wagner et al., Scanning 27 (2005) 298.

[7] Š. Mikmeková et al., Materials Transactions 51 (2010) 292.

[8] L. Frank, I. Müllerová, Ultramicroscopy 106 (2005) 28. 
[9] This research is supported by the Czech Science Foundation under grant P108/11/2270 and by the GAASCR project no. IAA100650902.
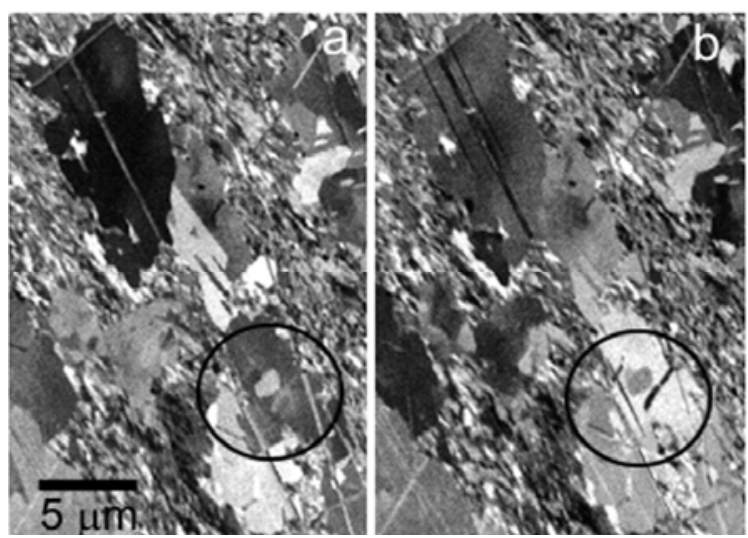

FIG. 1. Ultrafine grained $\mathrm{Cu}$ imaged in the $\mathrm{CL}$ mode at $2 \mathrm{keV}$ (primary energy $6 \mathrm{keV}$ ): untilted sample (a), sample tilted to $1.4^{\circ}$ (electron impact at $2.4^{\circ}$ ) (b); encircled area of inversions in contrast of twins and sub grains.

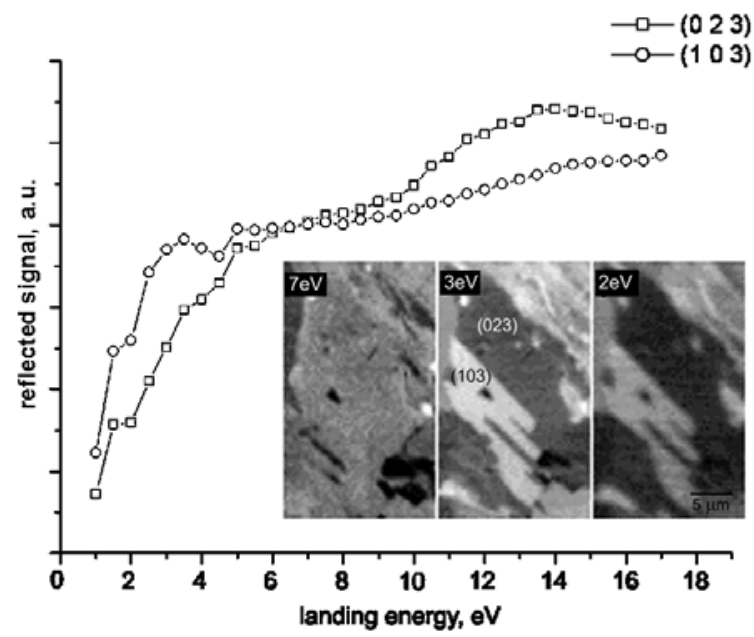

FIG. 3. Very low energy reflectance curves for two grains of an ultrafine grained $\mathrm{Cu}$ with selected micrographs illustrating development in the grain contrast.

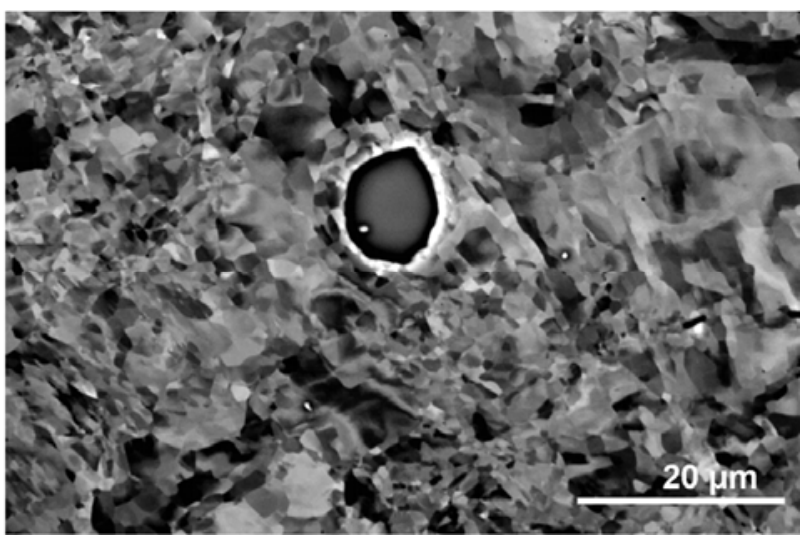

FIG. 2. Hot-rolled ferritic steel imaged in the $\mathrm{CL}$ mode at $5 \mathrm{keV}$ (primary energy $10 \mathrm{keV}$ ) with contrast variations inside grains revealing internal strain owing to heavy deformation.

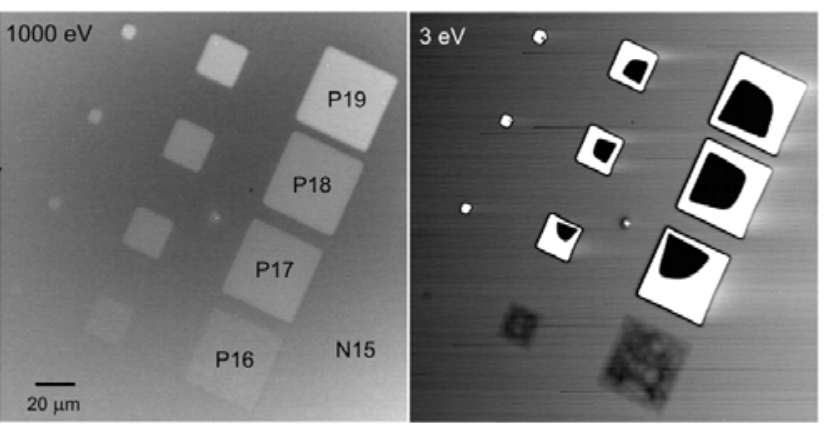

FIG. 4. Boron doped p-type patterns in n-type Si (100) substrate imaged in the CL mode with secondary electrons (left) and with BSE in the EBIV contrast (right). 